\title{
Building Normal ECG Models to Detect Any Arrhythmias Using Deep Learning
}

\author{
Keiji Gyohten ${ }^{1}$, Shota Hori $^{1}$, Hidehiro Ohki ${ }^{1}$, Toshiya Takami ${ }^{1}$, Noboru Sato ${ }^{2}$ \\ ${ }^{1}$ Faculty of Science and Technology, Oita University, Oita, Japan \\ ${ }^{2}$ Keiwakai Healthcare Group, Oita, Japan
}

\begin{abstract}
In the detection of arrhythmia using deep learning, most methods train neural networks to categorize input electrocardiogram (ECG) signals into typical arrhythmia signals using annotated training ECG data. Such methods can neither detect unknown arrhythmias nor explain why the signals are considered as arrhythmic. To detect arrhythmia automatically, this study proposes a method that learns normal ECG signals and can explain the assessment for the signals clearly. Our method builds a model of normal ECG signals using a convolutional neural network and long short-term memory. The model inputs normal ECG signals and performs training to predict the succeeding normal ECG signals. If an abnormal ECG signal is provided, the model predicts the succeeding ECG signal far from the actual ECG signal and can assess whether the input is abnormal. This means that our method can judge any arrhythmia because it requires no prior knowledge regarding the annotations. Experimental results confirm that the proposed method can detect arrhythmias appropriately without learning them. Furthermore, we propose a GUI that can display the location where abnormality is suspected to the user.
\end{abstract}

\section{Introduction}

In recent years, research regarding deep learning technology has progressed significantly in the field of machine learning. Because this technology can potentially result in breakthroughs in pattern recognition problems that are difficult to solve previously, deep learning techniques have been applied to various fields. For example, in the medical field, deep learning technology has been applied to image diagnosis [1] and disease diagnosis from electronic medical records [2].

The application of deep learning techniques to electrocardiographic analysis has been actively investigated [3-6]. In particular, the study in [7] indicated that the proposed method can yield diagnostic results comparable to those obtained by the average cardiologist. The application of deep learning techniques to electrocardiogram (ECG) analysis is likely to be attempted in various manner in the future.

In conventional deep learning techniques for ECG analysis, normal ECG waveforms and typical arrhythmia waveforms are trained by neural networks using supervised learning. In this case, the trained network detects abnormal waveforms in the ECG signal and only classifies them into typical arrhythmia types. It is difficult for such methods to detect untrained arrhythmias as suspected abnormalities and to explain why they are regarded as abnormal. In the future, as research pertaining to ECG analysis using deep learning technology progresses, we will require techniques that can detect not only typical arrhythmias, but also suspected unlearned arrhythmias. This technique should be able to explain the reasons for suspected arrhythmias.

Herein, we propose a method to generate a normal waveform model by learning only normal ECG signals and to acquire waveforms that do not fit the model as abnormal. The purpose of this study was to investigate the possibility of automatically detecting unknown arrhythmias by learning only normal waveforms, rather than learning the relationships between typical arrhythmias and their types. In addition, our method can present portions that differ from the normal waveform to explain why arrhythmia is suspected. The proposed method combines a convolutional neural network (CNN) and long short-term memory (LSTM), which are typically used for image recognition and time-series data analysis, respectively, to construct a model that represents normal waveforms. Through experiments, we demonstrate the performance obtained from using our method without learning the relationships between typical arrhythmia waveforms and their types. Furthermore, we propose a GUI that presents users with parts assessed as abnormal.

\section{Proposed method}

The proposed method builds a model that can predict a future wave from those in a certain period in the ECG signal by learning the normal waveforms in advance. If the predicted and actual waves match, then the ECG signal can be considered normal. Meanwhile, if the predicted wave differs significantly from the actual wave, 


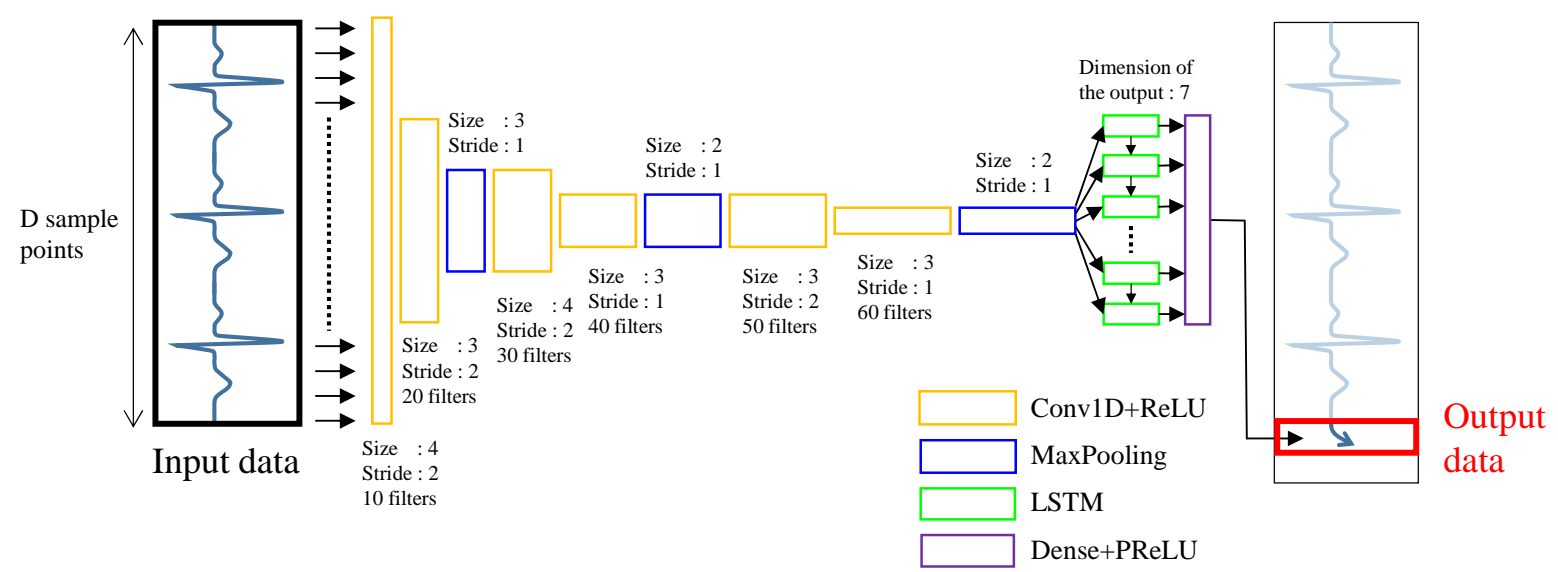

Figure 1. The proposed model for predicting normal ECG waveform.

it can be interpreted that abnormality is present at that part. This method can present both the predicted and actual waveforms simultaneously on the GUI, providing users with a visual indication of the location of anomalies.

\subsection{Model for predicting ECG waveforms}

The input/output data used in the proposed method and the model for predicting ECG waveforms are shown in Figure 1. The proposed model is populated with trimmed waveforms from an ECG section comprising D sample points that contain several $\mathrm{R}$ waves. The output of this model is the height of the wave following the waveform in this section. By comparing the wave height predicted by the model with the actual wave height, the proposed method determines whether the ECG is normal or abnormal.

As shown in Figure 1, the proposed ECG waveform prediction model is composed of a CNN and LSTM. The value of each hyperparameter was determined based on basic experiments.

\subsection{Assessment of abnormal waveform}

The input and output data to the model shown in Figure 1 are shown in detail in Figure 2. Our method assumes that each waveform in the ECG signal corresponds to a section represented by $\mathrm{W}$ sample points centered on an $\mathrm{R}$ wave. To analyze the waveforms of this section, the proposed method analyzes $\mathrm{W}$ pairs, each of which comprises a D-dimensional input vector representing the ECG wave immediately before a sample point and the output value representing the height of the ECG wave at the sample point.

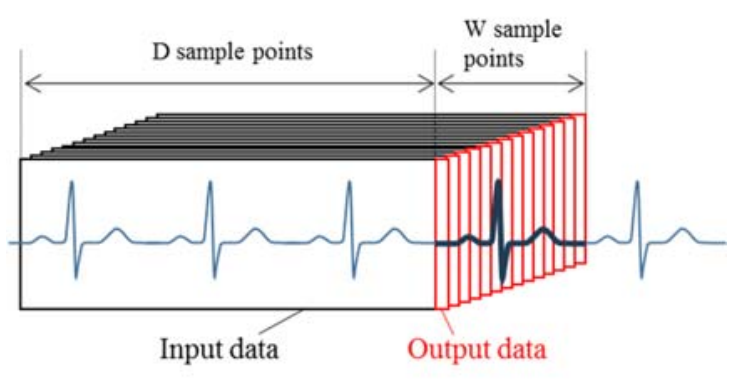

Figure 2. Input and output data for the proposed model.

The proposed method learns only the normal ECGs without learning arrhythmia and generates a normal ECG waveform model. The user selects the parts that are considered to be normal from the ECGs, eliminates them, and uses them as learning data. The learning data are the set of $\mathrm{W}$ pairs of the input vector and output values corresponding to the waveforms that are regarded as normal. These learning data are categorized into training and validation data, and the proposed model is trained using the training data. In this training, if the error between the model-predicted and actual wave height is not improved after a certain number of times using the validation data, early stopping is applied to terminate the process. At that time, the mean and standard deviation of these errors are calculated as the evaluation criteria for determining whether an ECG waveform to be analyzed is normal or abnormal.

When assessing whether a waveform is normal, our method feeds $\mathrm{W}$ pairs of input vector and output values related to this waveform to the trained model and predicts the wave heights. If the error between this predicted wave height and the actual one is more than three times the standard deviation obtained in advance, this actual wave height is regarded as abnormal. The waveform to be 
analyzed is regarded as abnormal if the ratio of the number of sample points whose wave heights are determined to be abnormal to $\mathrm{W}$ sample points that represent this entire waveform is above a certain threshold. We name this ratio the abnormal waveform ratio.

\section{Experiments}

In this section, we explain the experimental results obtained using the proposed method. The proposed method was implemented on an Intel Xeon E5-2620 2.10 GHz CPU with 16.0 GB of RAM and a GeForce GTX760 GPU chip. The model for predicting ECG waveforms was implemented using Keras and Tensorflow in Python. The GUI of this system was implemented using wxPython.

\subsection{Performance evaluation}

In the experiment, the ECG signals of 42 patients from the MIT-BIH arrhythmia database were used. In this database, the RR interval corresponds to approximately 300 sample points. Therefore, the number of sample points $\mathrm{W}$ corresponding to the length of each waveform in the ECG described in Section 2.2 was set to 300. As described in Section 2.1, D, which represents the number of sample points in the ECG signal to be input, was set to 900 to process an ECG signal containing several R waves in the proposed method. From the ECG signals of 42 patients, 500 waveforms annotated with normal heartbeats were obtained. Furthermore, 300, 50, and 150 waveforms among the 500 waveforms were used as training data, validation data, and test data, respectively.
As shown in Figure 2, because one waveform is composed of $\mathrm{W}$ pairs of input vectors and output values, the training data described above, for example, are composed of 90000 pairs of input vectors and output values. The validation data were used to apply early stopping termination when training the model. To confirm whether the normal ECG waveform model learned by the proposed method can assess whether an arrhythmia is abnormal, waveforms annotated with typical arrhythmias were obtained from the ECG signals of 42 patients. As typical arrhythmia test data, 150 waveforms each of left bundle branch block beats, right bundle branch block beats, atrial premature beats, premature ventricular contractions, and fusion of ventricular and normal beats were obtained.

To evaluate the performance of the proposed method, we constructed ROC curves representing changes in true and false positive rates obtained by varying the threshold of the abnormal waveform ratio described in Section 2.2. Figure 3 shows the ROC curve constructed by applying the proposed method to 150 waveforms annotated with arrhythmia and 150 normal waveforms; furthermore, the threshold of the abnormal waveform ratio was changed. Because the difficulty in diagnosis varies by the arrhythmia type, the ROC curve for each arrhythmia type was constructed. Desirable results were obtained for arrhythmias that were easy to diagnose, such as premature ventricular contraction, whereas the overall diagnostic performance was significantly inferior to that of conventional methods such as those presented in [7]. However, it is noteworthy that the proposed method does not learn all types of arrhythmia waveform. This indicates that the proposed method can diagnose ECG waveforms

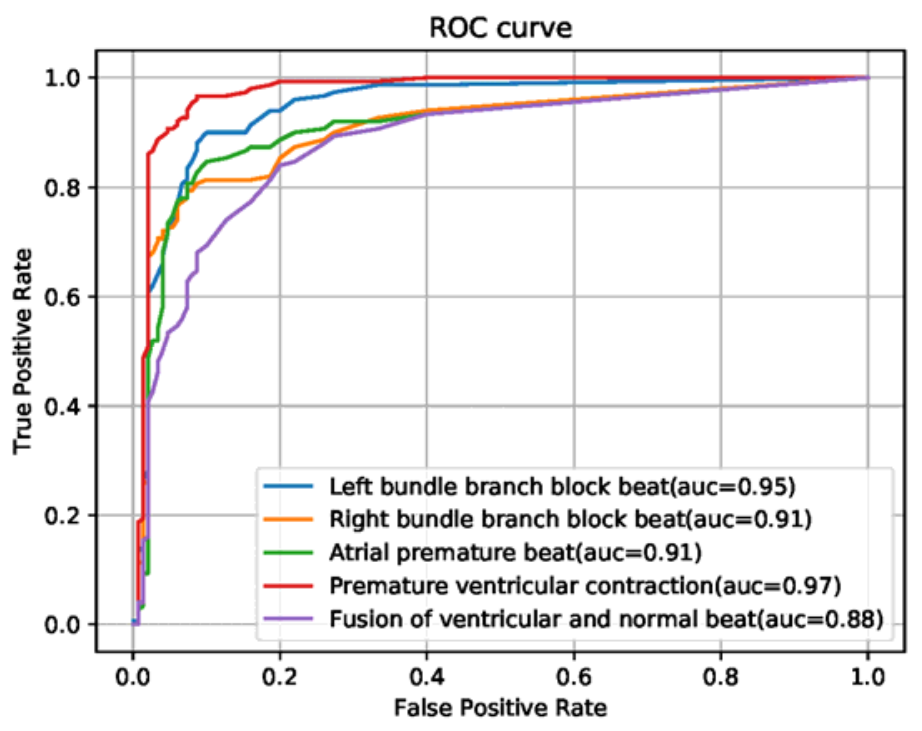

Figure 3. ROC curve obtained by applying the proposed method. 
that differ slightly from normal ECG waveforms as suspected arrhythmias. In the future, the proposed method should be improved based on the opinions of cardiologists.

\subsection{GUI}

The GUI currently under development is shown in Figure 4. One of the purposes of the GUI is to display the locations of suspected anomalies to the user. Furthermore, this GUI displays the predicted waves at the sample points where the actual wave height is regarded as abnormal by the method described in Section 2.2. This allows the user to determine the location at which the deviation from the normal waveform may occur. In the future, we plan to improve such information presentation on the GUI based on the opinions of cardiologists.

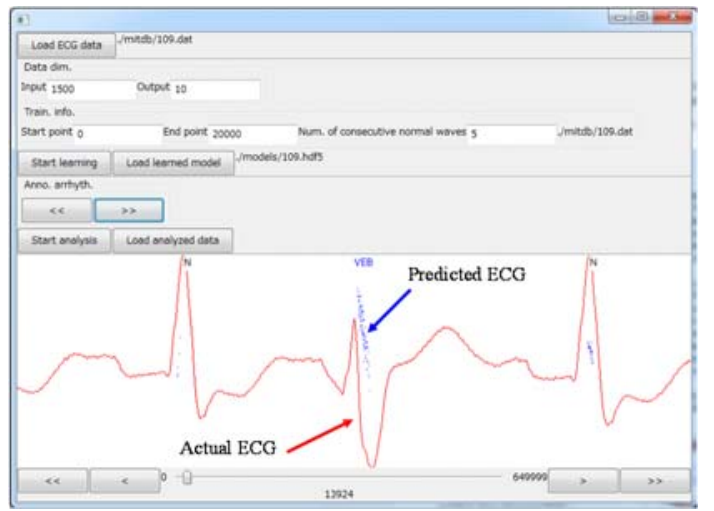

Figure 4. GUI of proposed method.

\section{Conclusions}

Herein, we proposed a method to construct a normal waveform model by learning only normal ECG signals and to assess waveforms that do not fit the model as abnormal. Experimental results showed that the performance of the proposed method was inferior to those of existing methods using supervised learning of arrhythmia waveforms by training neural networks. However, we successfully demonstrated the possibility of detecting arrhythmias by merely learning normal ECG signals. This may results in the diagnosis of waveforms that are not typical of arrhythmias and difficult to determine in terms of normality. In this context, we developed a GUI that displays the location of anomalies. We believe that this can assist doctors in diagnosis by presenting areas that do not suggest normal ECG signals.

Our current method can only assess whether an ECG signal is normal or abnormal. We will improve this method to classify arrhythmias by expressing the difference between a waveform diagnosed as abnormal and a normal waveform as a feature vector and then clustering these feature vectors. In addition, we will improve the GUI to support the diagnosis of arrhythmias based on the advice of cardiologists.

\section{Acknowledgments}

This work was supported in part by Artificial Intelligence Research Promotion Foundation.

\section{References}

[1] Lee H, Yune S, Mansouri M, et al. An explainable deeplearning algorithm for the detection of acute intracranial haemorrhage from small datasets. Nat Biomed Eng 2019; 3: 173-182.

[2] Liang $\mathrm{H}$, Tsui BY, Ni H, et al. Evaluation and accurate diagnoses of pediatric diseases using artificial intelligence. Nat Med. 2019; 25(3): 433-438.

[3] Kiranyaz S, Ince T, Hamila R, Gabbouj M. Convolutional neural networks for patient-specific ECG classification. Conf Proc IEEE Eng Med Biol Soc. 2015: 2608-2611.

[4] Zihlmann M, Perekrestenko D, Tschannen M. Convolutional recurrent neural networks for electrocardiogram classification. Computing in Cardiology (CinC) 2017: 1-4.

[5] Warrick P, Nabhan HM. Cardiac arrhythmia detection from ECG combining convolutional and long short-term memory networks. Computing in Cardiology (CinC) 2017: 161-460.

[6] Yildirim Ö. A novel wavelet sequence based on deep bidirectional LSTM network model for ECG signal classification. Comput Biol Med. 2018; 96: 189-202.

[7] Hannun AY, Rajpurkar P, Haghpanahi M. et al. Cardiologist-level arrhythmia detection and classification in ambulatory electrocardiograms using a deep neural network. Nat Med 2019; 25: 65-69.

Address for correspondence:

Keiji Gyohten

Faculty of Science and Technology, Oita University

700 Dannoharu, Oita 870-1192, Japan

gyohten@oita-u.ac.jp 